International Journal of Biological Sciences

ISSN 1449-2288 www.biolsci.org 2007 3(7):446-454

CIvyspring International Publisher. All rights reserved

Research Paper

\title{
Immunoregulatory role of intestinal surfactant-like particles during Salmonella typhimurium infection
}

\author{
M. Hanief Sofi, ${ }^{1,2,3}$, Archana Bhatnagarr,2, Saveeta Sapra ${ }^{1}$, Akhtar Mahmood² and Sidhartha Majumdar $^{1}$ \\ 1. Department of Experimental Medicine \& Biotechnology, Postgraduate Institute of Medical Education and Research \\ (PGIMER), Chandigarh, India \\ 2. Department of Biochemistry, Panjab University, Chandigarh, India \\ 3. Department of Microbiology and Immunology, University of Michigan School of Medicine, Ann Arbor, MI 48109, USA
}

Correspondence to: M. Hanief Sofi, PhD, Department of Microbiology and Immunology, University of Michigan School of Medicine, Ann Arbor, MI 48109. Email: mhanief@umich.edu; Ph: 734-647-7579

Received: 2007.05.27; Accepted: 2007.11.01; Published: 2007.11.12

Surfactants like particles (SLP) are secreted by Intestinal epithelium. These particles have the ability to lower surface tension of intestinal epithelial cells and contain small amounts of surfactant specific proteins A, B, and D. In the intestinal lumen they are known to function as lubricants and/or as a vehicle to deliver digestive enzymes to the luminal fluid. These particles have been found to have the ability in binding of uropathogenic E.coli. But their immunological function is not known. The present study was designed to assess the role of the SLP in the regulation of immune response during Salmonella $(S)$ typhimurium infection using a rat an enteric model. The animals were divided in four different groups including control (PBS), rats fed fat diet (corn oil), rats fed fat diet followed with S. typhimurium infection and rats with S. typhimurium infection alone. The Peyer's patches (PP), intraepithelial (IE) and lamina propria (LP) mononuclear cells were isolated from the above-mentioned groups. These mononuclear cells were then incubated in presence of S. typhimurium lysate alone, SLP alone and S. typhimurium lysate and SLP together. T cell markers CD4 and CD8, cytokines mainly pro-inflammatory ones including IFN- $\gamma$, TNF- $\alpha$, IL-12 etc were studied under such conditions. In addition histological studies were also carried out under these conditions. We report in this study that SLP plays an important role in modulating the cytokine level during infection. The pro-inflammatory cytokines were found significantly reduced in SLP induced diet along with the infection group compared to the infection group alone. Histopathological studies revealed the breakdown of duodenal villi after infection while only broadening of villi was observed in rats given corn oil induced SLP along with infection. These results suggested an important immuno-modulatory role for SLP during Salmonella infection.

Key words: Surfactant Like particles, microbial invasion, Salmonella typhimurium, immune response, cytokines

\section{Introduction}

The intestinal epithelium constitutes the major mechanical barrier between luminal pathogens and the mucosal immune system. An intact epithelial barrier function is thus critical to protect the host organism from invading luminal pathogens, restricting microbial entry and propagation. In turn, enteroinvasive bacteria, including Salmonella, are capable of traversing this barrier by infecting epithelial cells and ultimately translocating to the mucosal and submucosal layers. Eventually, enteroinvasive pathogens will enter the intestinal microcirculation, resulting in systemic infection and causing septic illness. The innate immune system plays an essential role in the early responses to Salmonella infections may be enough to control progression to disease [1]. Following ingestion, the pathogen is able to colonize the intestinal tract and penetrate the intestinal epithelium to ultimately gain access to systemic sites, such as the liver and spleen, through lymphatic and blood circulation. S. typhimurium invasion genes are necessary for bacterial invasion of intestinal epithelial cells and are thought to allow Salmonella to enter and cross the intestinal epithelium during infection by manipulation of the host cytoskeleton [2]. More recent findings have shown that CD18 positive dendritic cells (DC) are able to take $S$. typhimurium across the intestinal mucosa and possibly carry the bacteria to the spleen and liver [3] In mice, S. typhimurium is rapidly detected in lamina propria DCs, which may acquire Salmonella through transepithelial dendrites from the subepithelial space, or apoptotic intestinal epithelial cells [4]. Recently it has been demonstrated that $S$. typhimurium infection of T84 intestinal epithelial cell monolayers leads to modulation of the expression and/or distribution of various proteins that comprise the epithelial cell tight junction in a different fashion. On the basis of the pathophysiological consequences of these alterations, an additional mechanism is possible that $S$. typhimurium may employ to breach the intestinal epithelial barrier to become systemic and promote disease [5]. Although the innate immune system is the primary line of defense against Salmonella 
infections, it is clear that the acquired immune system is important for clearing the infection as well as for providing effective protection to subsequent challenge with related Salmonella strains.

Intestinal epithelium secretes unique lipoprotein lamellar structures, which are rich in phosphatidyl choline and intestinal alkaline phosphatase (IAP) and have the buoyancy characteristics similar to lung surfactants. These lamellar structures are called Surfactant like particles (SLP) [6]. These particles have the ability to lower surface tension of intestinal epithelial cells and contain small amounts of surfactant specific proteins $A, B$, and D. In the intestinal lumen they seem to cover the surface of the cell, where they may act as lubricants and/or as a vehicle to deliver digestive enzymes to the luminal fluid [6]. These particles have been found to have the ability in binding of uropathogenic E.coli [7]. Also the decrease in SLP in active duodenal ulcer or duodenitis suggesting that SLP is consumed by inflammatory process or that the synthesis / secretion of the SLP was altered. Such a change in SLP content might play a part in the pathogenesis of duodenal ulcer disease [8]. Because the amount of fresh human tissue available for SLP isolation is quite limited, we sought an animal model to further characterize the role of SLP during infection. In this paper, we have reported that SLP plays an important role in modulating the cytokine level during infection. In addition it has also seen in the present study by histological analysis that the epithelial layer is disrupted in infection as reported previously also but is protected when provided with Salmonella infection along with SLP induced corn oil suggesting the important immunomodulatory role of SLP during infection.

\section{Materials and Methods}

\section{Laboratory animals}

In the present study, Sprague-Dawley rats with body weight ranging from 150-180 gm were procured from the central animal house PGIMER, Chandigarh. They were housed in individual cages, acclimatized to the laboratory conditions and fed with standard pellet diet and water ad libitium. They were also administered a course of broad-spectrum antibiotics and anti-helminthic drugs to take care of previous bacterial or parasitic infections. For the following one-month, the rats were strictly fed on autoclaved diet and water. The animals were divided into four groups: Control, animals fed fat diet only, animals fed fat diet and infection and animals on infection only. Each group had ten (10) rats. This study was approved by institute animal use and care committee and dean's doctoral committee.

\section{Bacterial strain}

Salmonella typhimurium was used as an infecting agent and was maintained in the laboratory in nutrient broth medium. This pathogenic, invasive strain (ATCC 14028s) was obtained from MTCC center of Institute of Microbial Technology, Chandigarh, India. The whole cell lysate of Salmonella typhimurium used in some experiments was prepared by sonication of the bacteria for 2-3 minutes. The protein content of the lysate was estimated by Lowry's method [9].

\section{Infection protocol}

The bacterial count for infection was adjusted by standard Colony forming unit (CFU) determination. Briefly, S. typhimurium was grown aerobically in nutrient broth overnight at $37^{\circ} \mathrm{C}$. Bacterial cultures were harvested, washed and suspended in normal saline. Serial 10 fold dilution of known number of bacteria / $\mathrm{ml}$ was made with sterile normal saline and from each dilution $10 \mu \mathrm{l}$ of bacterial inoculum was incubated in nutrient broth (Difco) and was plated out and incubated at $37^{\circ} \mathrm{C}$ overnight. The colonies were counted after $\mathrm{O} / \mathrm{N}$ incubation and $\mathrm{CFU}$ was calculated. In the log phase of bacteria, the number of cells counted after plating was $10^{7}$ cells $/ \mathrm{ml}$ of broth. This was taken as a standard for infecting the rats under different conditions.

\section{Mode of Infection}

Rats were given $0.25 \mathrm{ml}$ of a solution of $0.2 \mathrm{M}$ $\mathrm{NaHCO}_{3}$ orally through gastric gauges, to neutralize acidity [10] and after waiting for 5 minutes, $S$. typhimurium $\left(2 \times 10^{7} \mathrm{CFU} / 1 \mathrm{ml}\right)$ was administered [11]. Animals had free access to water but were not allowed to eat for $24 \mathrm{~h}$ before giving infection as well as before the sacrifice.

\section{Isolation of Surfactant like particles from rat intestine}

The SLP were isolated by the method of Eliakim et al. (1989) [3]. Briefly, male Sprague-Dawley rats fasted overnight were fed with corn oil intragastrically and then sacrified after 5-7 hrs of feeding. The intestine was opened longitudinally, and the mucosal surface was lightly scraped using whattman no.3 paper and suspended in solution A [5mM Tris (hydroxy methyl) amino methane buffer, $\mathrm{pH} 7.4$, containing $5 \mathrm{mM} \mathrm{CaCl}_{2}$ ].

Gradient centrifugation: SLP were isolated from the light mucosal scrapings by passing through a gradient that separated membrane bound phosphatase (density 1.07-1.08g/L) from free IAP (density $1.057 \mathrm{~g} / \mathrm{L}$ ). The mucosal scrapings from the fat fed group were fractionated on continuous $\mathrm{NaBr}$ gradients (0.49-1.49 $\mathrm{M}$ in solution A) as described for the isolation of human lung surfactant [6]. A visible white band of density 1.07-1.08g/L was formed against clear solution after centrifugation at $100,000 \mathrm{xg}$ for $16 \mathrm{~h}$ at $5^{\circ} \mathrm{C}$ using an SW-41 rotor (Beckman instruments). These conditions were identical to those used by Eliakim et al (1989) [3] to isolate rat intestinal SLP. One-milliliter fractions were removed from the top of the gradient by siphoning and used for biochemical analysis. Fractions 3 and 4, exhibiting maximal IAP activity, were pooled from several gradients, dialyzed and concentrated using an Amicon filter (XM-10) assembly (Millipore Corp., Bedford, MA). This SLP isolated from rats which were given a fat diet only, was used throughout the experiments. The SLP from a control group of rats 
not given a fat diet was isolated separately and used as control throughout the experiments.

\section{Isolation of Peyer's patch (PP), intraepithelial (IE) and lamina propria(LP) mononuclear cells}

The methods of Davis and Parrot [12] and Lyscom and Bruetion [13] were used for isolation. Briefly, rats were anaesthetized with anesthetic ether and killed by cervical dislocation. The small intestine was immediately removed, flushed with citrate buffer and slit open. The PP was carefully dissected out. The cells were released from the PP by scraping with a scalpel. After Peyer's patches were removed, the intestines were opened longitudinally washed in HBSS. The intestine was then opened longitudinally and cut laterally into small pieces $(0.5-1 \mathrm{~cm})$. These pieces were put into a flask containing citrate buffer/BSA and were incubated for 30 minutes at $37^{\circ} \mathrm{C}$ in a shaking water bath, followed with vigorous shaking for 15 seconds on vortex. The supernatant fluid was removed, replaced and the shaking process repeated till the supernatant fluid was clear and the IELs were collected. The tissue fragments were incubated for an additional 30 minutes, shaking at $37^{\circ} \mathrm{C}$ in HBSS with $1 \mathrm{mM}$ DTT and $5 \mathrm{mMM}$ EDTA, and the remaining IEL/enterocytes were discarded. The remaining tissue was incubated for 20 minutes with stirring in presence of collagenase to facilitate the disruption of the gut pieces. Then the supernatant was collected and repeated the same till the supernatant was found clear. The PP, IE and LP mononuclear cells in citrate buffer /BSA were filtered through cotton gauze to remove coarse clumps and subsequently passed through cotton wool filters. The cells were further purified by Ficoll isopaque. Cell recovery and viability was determined by trypan blue exclusion $(0.05 \%$ in saline) using a haemocytometer counting chamber.

\section{Cell surface staining for evaluating $\mathbf{T}$ cell numbers}

Monoclonal anti-rat CD4 or CD8- FITC antibody (Serotec, U.K.) was added to $2 \times 10^{5}$ cells and incubated for one hour at $4^{\circ} \mathrm{C}$. At the end of incubation tubes were centrifuged and the pellet was resuspended and washed twice with PBS. Finally the cells were fixed with $0.5 \mathrm{ml}$ of fixative $(0.5 \%$ Paraformaldehyde in PBS) for 30 minutes at $4^{\circ} \mathrm{C}$. Then the sample was analyzed on FACScan by using Cell Quest software (BD, Becton Dickinson).

\section{Cytokine production}

The cells $\left(2 \times 10^{6}\right.$ cells/well) from different groups were cultured in 48 well plates with Salmonella typhimurium lysate $(6 \mu \mathrm{g})$ and surfactant like particles $(6 \mu \mathrm{g})$ alone and together. Supernatants were harvested after 48 hours and stored at $-70^{\circ} \mathrm{C}$ until testing. IL-2, IL-4, IL-10, TNF- $\alpha$, IFN- $\gamma$ and IL-12 were measured in these supernatants by a standard ELISA method. Monoclonal antibodies to the rat cytokines IL-4, 10, IFN- $\gamma$, TNF- $\alpha$ and IL-12 antibody (Serotec, UK) were added and incubated for $2 \mathrm{hrs}$ at $37^{\circ} \mathrm{C}$. After washing, the plate was incubated with blocking buffer for 2hrs at $37^{\circ} \mathrm{C}$ and later washed with wash buffer. Horseradish peroxidase enzyme (HRP) labeled secondary antibody was then added to the wells for 2 hours and after proper washing, the freshly prepared substrate was added and OD was taken at $492 \mathrm{~nm}$. The standard graph was plotted using recombinant cytokines (Serotec, UK).

\section{Histopathological Examination}

For histopathological examination, full thickness samples from the duodenum segment of different groups of rats were removed and fixed in 10\% neutral buffered formalin. Paraffin embedding was performed and blocks were sectioned using standard methodology. Paraffin sections were stained with hematoxylin and eosin (H\&E) and the slides were examined for any local inflammatory changes under light microscope.

Electron microscopy of the rat duodenal epithelium was carried out by using method of Collet (1979) [14]. Briefly segments of the tissue fractions were fixed for $1 \mathrm{hr}$ in $2.5 \%$ glutaryldehyde in $0.1 \mathrm{M}$-cacodylate buffer at room temp. and then post fixed in $1 \% \mathrm{OSO}_{4}$ in the same buffer for $1 \mathrm{hr}$ at $4^{\circ} \mathrm{C}$, followed by several changes of $100 \%$ alcohol at room temp. The pellets were dehydrated in acetone and subsequently embedded in pure epon. Ultrathin sections were cut in the LKB loba ultra microtome, stained with uranyl acetate and lead citrate, finally examined by transmission electron microscopy (Zeiss, 906 Germany).

\section{Results}

M cells present in the epithelium overlying PP are well known to be targeted by different enteropathogens, thereby enabling their uptake into the intestinal mucosa. In addition, alternative routes of entry via $M$ cells outside of PP via lamina propria dendritic cells or by direct invasion of absorptive enterocytes have been proposed. The host has different ways to overcome the infection. Reports have shown that Salmonella disturbs the epithelial layers to cause the systemic infection. Previously it was also reported that SLP covers the surface of intestinal epithelial cells and it is possible that they might have some immunological role during infection. In the present study we asked if SLP on the epithelial surface layer has protective role during infection. To address this question, we did the histology of intestine from all these different groups mentioned in material and methods. As shown in figure 1A the control group has normal morphology and almost no or very less infiltration of pro-inflammatory cells. On the other hand, infected group as shown in figure 1D has extensive increase in the inflammatory cells, increased neutrophils and villi tips exhibited necrosis in the cross section. However the cross section from fat fed induced SLP along with infection (experimental group) showed increased fat vacuoles, increased inflammatory cells and a sub epithelial edema similar to that found in the fat fed group (1B), but no necrosis as shown in figure $1 C$. The proinflammatory cells were 
far less than from infection group alone. We asked whether the difference is more prominent at higher resolution. We used same set of groups and analyzed them by electron microscopy. As shown in figure 2, the transmission electron microscopic studies shows microvillus damage in the cross section of the rat duodenum given infection alone (figure 2D) while the microvilli from the fat fed infected group showed the shortening and broadening of microvilli, but not any damage to the villi $(2 \mathrm{C})$. These results suggested the protective role of fat induced SLP during infection. From electron microscopic examination, it is clearly shown the disruption of the microvilli from the infected group, which is not the case when SLP induced diet along with infection was given to these rats suggesting an important role of fat induced SLP during infection.
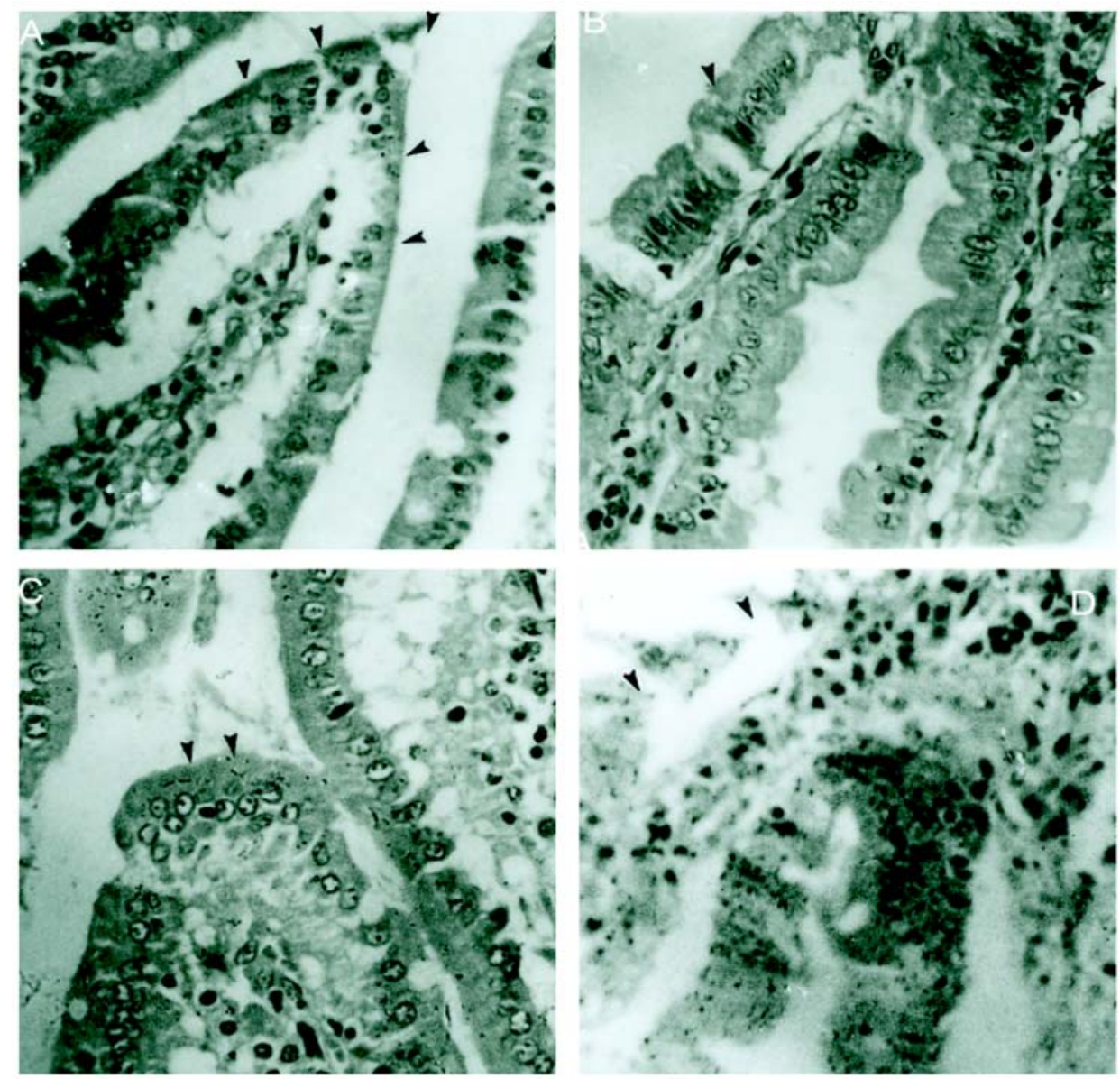

Figure 1. Histological sections of small intestinal villi shows no necrosis from S.typhimurium infected together with SLP induced diet. (A) Cross section of the normal small intestinal villi showing normal villus height. Arrows points to the normal pointed villus. (B). Cross section of the fat fed rat small intestinal villi showing edema in the lamina propria and broadening of villi. Arrows points to a broadened villus. (C) Shows fat deposited in lamina propria of the cross section of fat fed with S.typhimurium infected rat. (D) Demonstrates increased infiltration of neutrophils and also shows villus necrosis (arrow). Magnification x 40. 

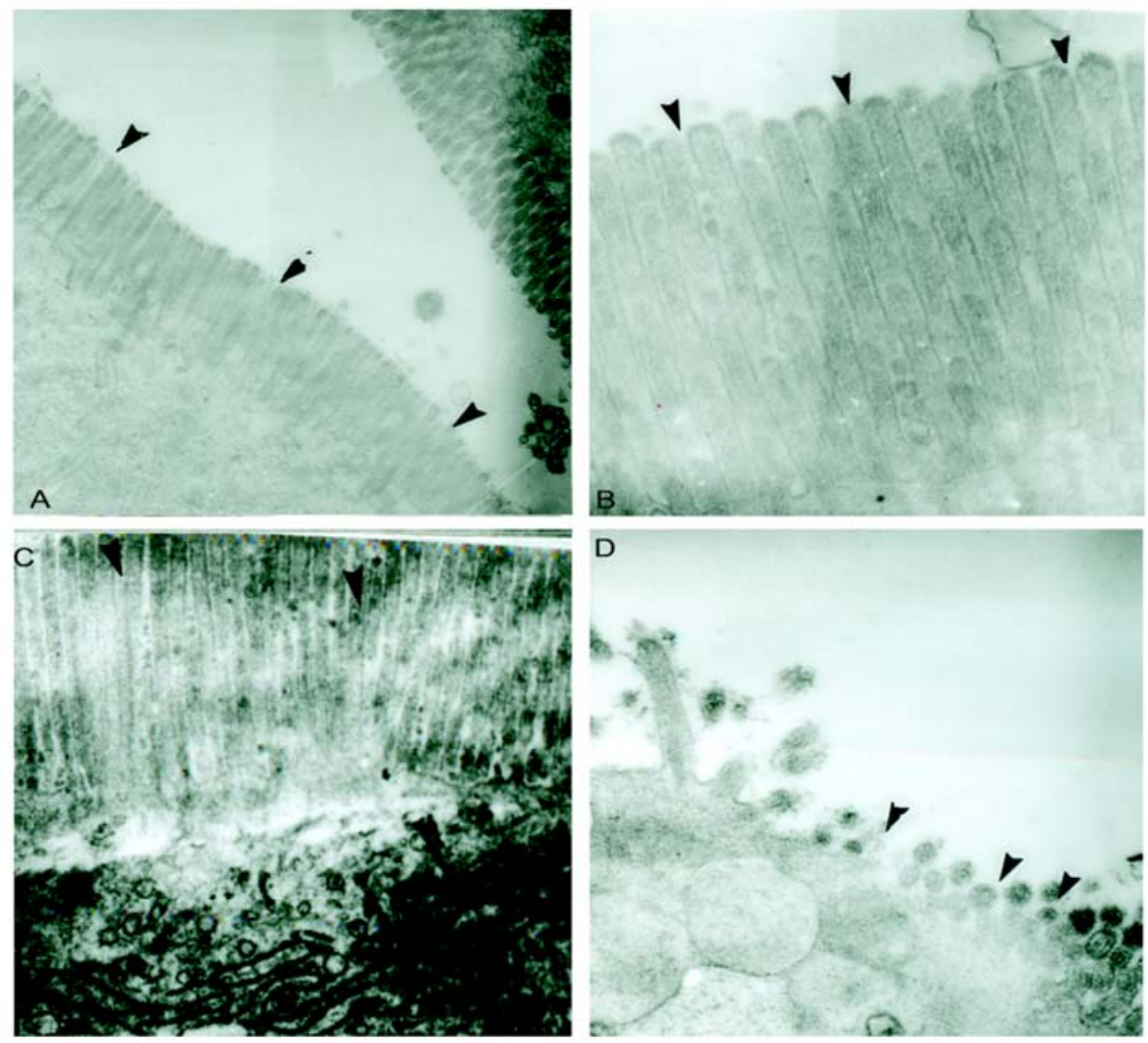

Figure 2. Electron microscopic studies of small intestinal villi shows microvilli damage from S.typhimurium infected group but was protected from S.typhimurium infected along with SLP induced group. (A) Shows electron micrograph of normal small intestinal villi. (B) Electron micrograph of small intestinal villi isolated from fat fed rat showed fat droplets and little broadening of villi. (C) Intestinal villi after fat feeding and S.typhimurium infection shows slight necrosis of the intestinal villi (D) Intestinal villi isolated from the rat given S.typhimurium infection shows major disruption of microvilli. The arrow points to such regions of micro villi. magnification 4646X.

\section{FACS analysis of $T$ cell surface markers from peyer's patch mononuclear cells}

Based on the histopathological results, we asked if there is any variation in the various surface molecules on various immune cells. To address this question, the mononuclear cells were separated from PP, IE and LP (by standard protocols) and were later analyzed for CD4 and CD8 T cell markers. Previous reports have shown that lung surfactants are well known to have immuno suppressive effects. The relative immunosuppressive effects of surfactant are likely to have an impact on the initial and subsequent interactions between the pathogen and the mononuclear cells. The SLP is known to have many similar functions to lung surfactants including surface tension reducing activity and have surfactant specific proteins including $\mathrm{A}, \mathrm{B}$ and $\mathrm{D}$, but unknown their immunological similarity if any. Previous reports have also shown that the decrease in SLP in active duodenal ulcer or duodenitis suggesting involvement of SLP under such conditions [10]. The next obvious question was to see if there is any variation in the cell composition especially CD4 and CD8, the two arms of the adoptive immune system from above mentioned groups. As shown in table 1, the FACS analysis of T cell markers on mononuclear cells from PP, IE and LP revealed that the number of $\mathrm{CD} 4^{+}$and $\mathrm{CD} 8^{+} \mathrm{T}$ cells was significantly higher after infection as compared to their respective controls $(\mathrm{p}<0.05)$. On the other hand the cell surface staining from corn oil induced SLP along with infection was not much different as compared to the controls. These results suggest that fat induced SLP modulates the T-cell response upon bacterial challenge.

Table 1. Analysis of T cell population in different conditions. The data is expressed percentage of cells positive for the respective fluorochrome tagged antibody.

\begin{tabular}{|l|l|l|l|l|}
\hline PPL & Control & Fat & Fat+infect. & Infection \\
\hline CD4+ & $13.5 \pm 1.0$ & $9.6 \pm 0.5$ & $16.7 \pm 1.2$ & $46.9 \pm 3.4$ \\
\hline CD8 & $3.5 \pm 0.0$ & $4.3 \pm 0.1$ & $13.6 \pm 0.0$ & $25.2 \pm 3.2$ \\
& & & & \\
\hline IEL & & & & \\
\hline CD4 & $14.1 \pm 1.7$ & $10.9 \pm 2.1$ & $17.8 \pm 2.1$ & $34.9 \pm 2.8$ \\
\hline CD8 & $12.3 \pm 2.2$ & $17.4 \pm 1.8$ & $34.7 \pm 3.3$ & $68.3 \pm 4.9$ \\
\hline LPL & & & & \\
\hline CD4 & $11.2 \pm 2.9$ & $12.6 \pm 1.3$ & $19.2 \pm 1.6$ & $33.7 \pm 3.8$ \\
\hline CD8 & $18.2 \pm 1.3$ & $14.4 \pm 0.9$ & $27.3 \pm 2.4$ & $39.2 \pm 3.3$ \\
\hline
\end{tabular}




\section{Cytokine production by PP mononuclear cells Stimulated with $S$. typhimurium lysate}

Once the infection dominates over the host, there is induction of various cytokines based on the status of infection. Previous reports have shown that during Salmonella infection, there occurs an induction of pro-inflammatory responses [15] To test the effect of SLP under such conditions. The PP, IE and LP mononuclear cells from groups mentioned in materials and methods were isolated and were then cultured for $48 \mathrm{~h}$ in presence and absence of bacterial lysate $(6 \mu \mathrm{g} / \mathrm{ml})$ alone and together with SLP and supernatant was analyzed for different cytokines by ELISA. The amount of lysate used was based on dose response curve. The purification of SLP was characterized based on its IAP activity, as well as protein lipid ratio as has been characterized in many published reports.

\section{Pro-inflammatory Cytokines}

The cells isolated from above mentioned groups were cultured in vitro in presence of bacterial lysate alone or together with SLP. As shown in figure 3, the levels of pro-inflammatory cytokines IFN- $\gamma$, TNF- $\alpha$ and IL-12 were found significantly increased during infection alone group from all different kind of cells (including PP, IE and LP) which was not the case with SLP induced diet along with the infection group. However when the cells were cultured invitro with SLP along with bacterial lysate (from corn oil induced SLP group), a significant decrease in the cytokines level was observed within this group as compared to cells cultured in vitro with bacterial lysate only. In addition cells purified (from fat induced SLP along with infection group) were cultured in presence of either lysate alone and lysate and SLP together, also showed a drastic

Figure 3. Effect of SLP on pro-inflammatory cytokines IFN- $\gamma$ and TNF- $\alpha$ induction. Peyer's patch mononuclear cells $\left(2 \times 10^{6} / \mathrm{ml}\right)$ isolated from rat intestine under different conditions (as described in material and methods) were incubated in presence of S.typhimurium lysate $(6 \mu \mathrm{g} / \mathrm{ml})$ alone or together with SLP $(6 \mu \mathrm{g} / \mathrm{ml})$ for $48 \mathrm{~h}$ at $37^{\circ} \mathrm{C}$. Supernatant was isolated and analyzed by ELISA for (A) IFN- $\gamma$ and (B) TNF- $\alpha$. These values are represented as mean \pm SD. Results are representative of three independent experiments.
A

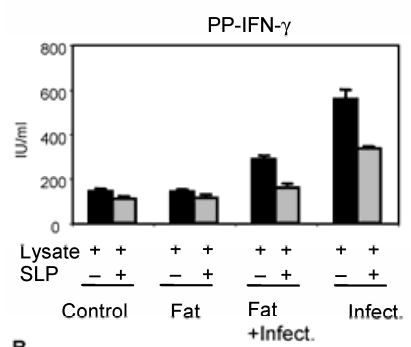

B
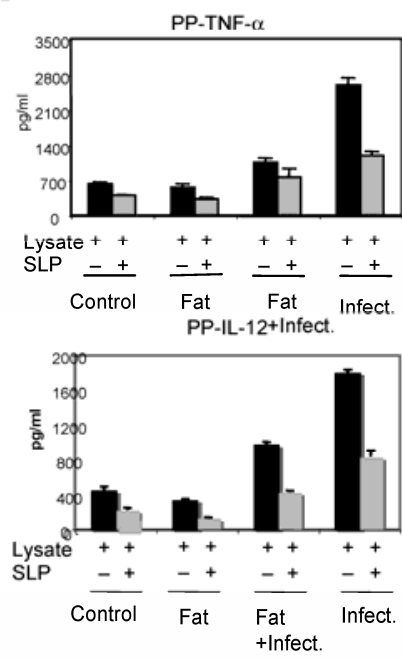

decrease in these pro-inflammatory cytokine when cultured together with SLP as compared to infection group alone. In addition the group where only infection was given in vivo and later cultured with SLP showed less inhibition than the previous groups suggesting induction of SLP in vivo is important to control the infection. No significant difference was observed on IL-2 in presence and absence of SLP (data not shown). These results clearly suggested that SLP modulates inflammatory response in-vivo during enteric infection, however mechanism of down regulation of pro-inflammatory signals by SLP during infection is not clear. In one of the studies carried out by Mahmood et al (2000) [16], it has been shown that SLP binds to the uropathogenic E.coli and prevents further activities of microorganism, is probably the same mechanism by which SLP prevents the binding of pathogen into the epithelial layer in vivo as well, an important step for pathogen to cause infection.

We further analyzed the effect of SLP on anti-inflammatory cytokines IL-4 and IL-10 by ELISA, as shown in Fig. 4, a significant increase in these cytokines were also observed from infection group. Interestingly, SLP induced diet along with infection group has shown reduced IL-4 cytokine levels but IL-10 cytokine levels were un-affected. The cytokine status from control group was either undetectable or at very low level (data not shown). The overall data showed here suggested the important immuno-modulatory role of SLP during infection.
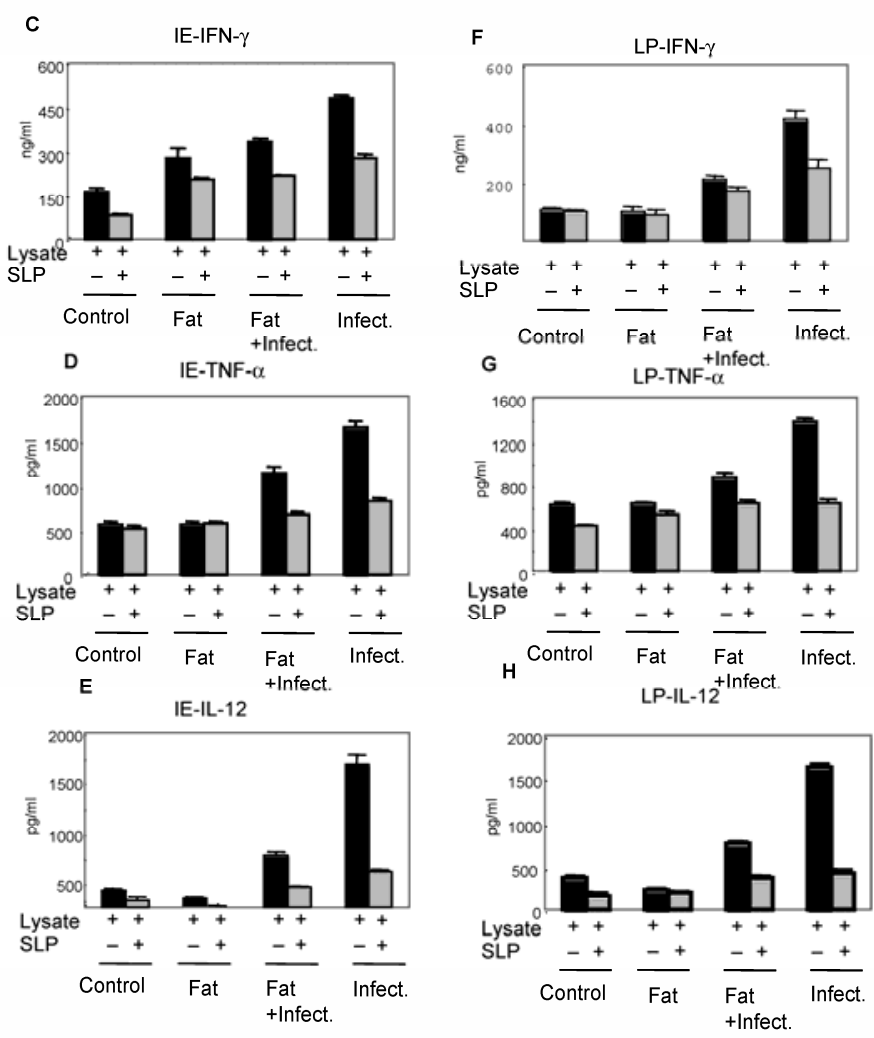

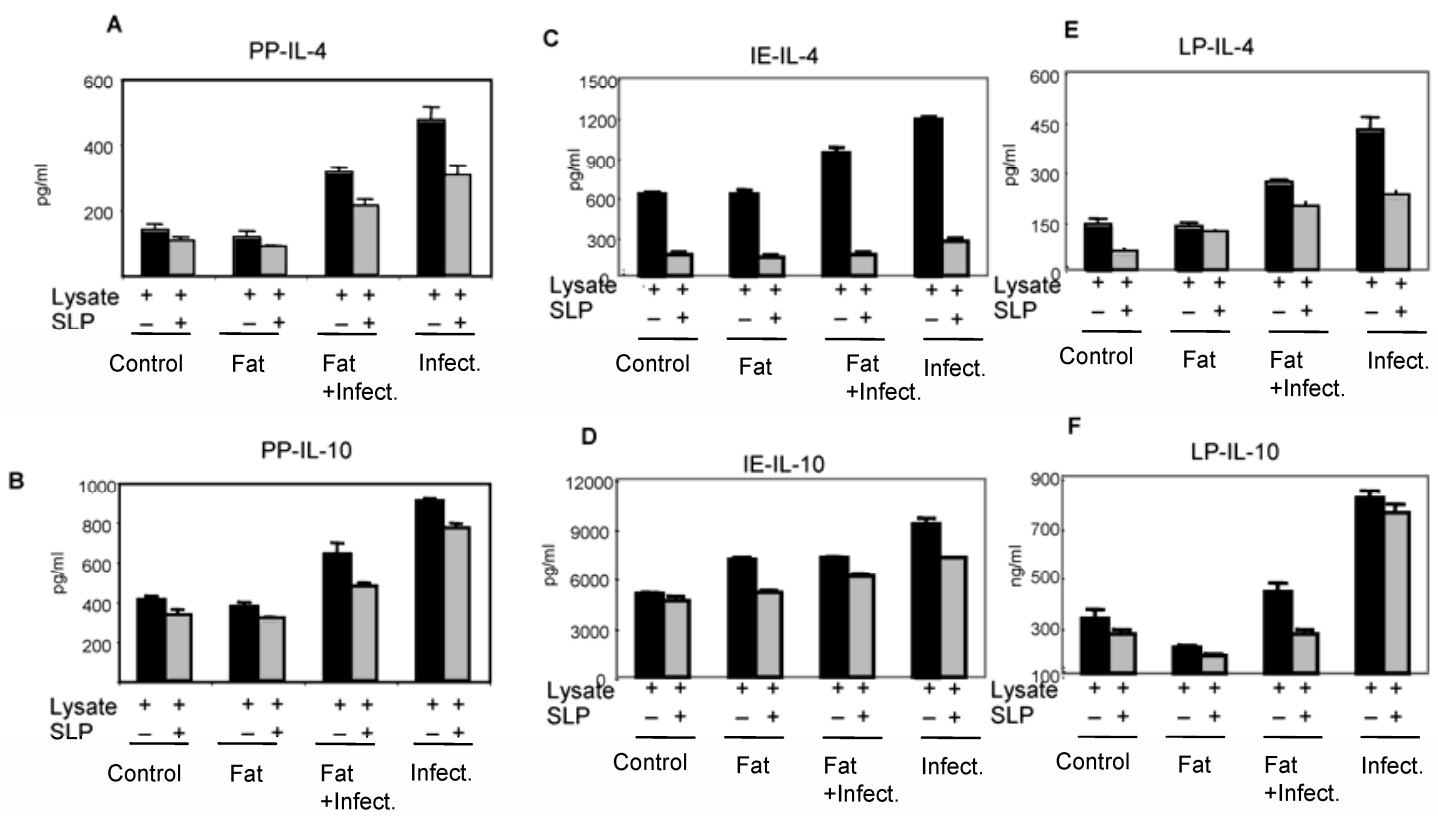

Figure 4. Effect of SLP on anti-inflammatory cytokines IL-4 and IL-10 induction. Peyer's patch mononuclear cells ( 2 X $\left.10^{6} / \mathrm{ml}\right)$ isolated from rat intestine under different conditions (as described in material and methods) were incubated in presence of S.typhimurium lysate $(6 \mu \mathrm{g} / \mathrm{ml})$ alone or together with SLP $(6 \mu \mathrm{g} / \mathrm{ml})$ for $48 \mathrm{~h}$ at $37^{\circ} \mathrm{C}$. Supernatant was isolated and analyzed by ELISA for (A) IL-4 and (B) IL-10. These values are represented as mean \pm SD. Results are representative of three independent experiments.

\section{Discussion}

In this study, we investigated the immuno-modulatory role of SLP on intestinal epithelium during S. typhimurium infection. A number of enteric pathogens are known to perturb the intestinal epithelial barrier by mechanisms, which may be unique for different pathogens [17]. It has also been demonstrated that the invasion of polarized human intestinal epithelial cells by $S$. typhimurium is associated with rapid molecular changes in the composition of tight junctional complexes leading to the loss of barrier function. The disruption of epithelial barrier integrity by Salmonella has pathological consequences by enhancing bacterial translocation across the epithelial barrier and fosters disease pathology by promoting migration of PMNs [5].

Dietary inputs influence the immune system directly by providing energy and nutrients needed by the host [18]. The SLP is known to be associated with the apical membrane of the cell and its immuno-modulatory function during infection has not been characterized. Present study has found an important immuno-regulatory role for SLP during infection. The original observations identifying surfactant material in the gastrointestinal tract described phospholipids, such as phosphatidylcholine and phosphatidylglycerol, adsorbed to the apical surface of the mucosa from the esophagus to the rectum in man [19], and its effects in preventing duodenal mucosal injury by acid as well as the participation of surface hydrophobicity and zwitterionic phospholipids of the mucus layer in this process. This lipid layer was postulated to function as a defensive barrier to protect the mucosal surface from both mechanical and chemical insult [20]. Thus, local accumulation of surface-active phospholipids may be an integral component of the cyto-protective mechanism usually induced by specific diets. SLPs have since been isolated from human small intestinal and colonic mucosa, and contain unique proteins not present in either MVM or basolateral membranes. One of these proteins is surfactant protein A. The presence of this protein could provide the intestine with a first line of defense against invasion by bacteria, since surfactant protein A is known to interact with bacteria in the lung and promote the clearance of these pathogens by macrophages [21].

A number of microorganisms have developed the capacity to invade the cells of the host and induce the immune system. Previous reports have shown that T cells play an important role during infection of intracellular pathogens by activating the microbicidal activity of macrophages by the secretion of IFN- $\gamma$ [22]. The up-regulation of pro-inflammatory cytokines are important to curb the infection. But on the other hand, the excess of pro-inflammatory responses is also known to be detrimental to the host. So these proinflammatory responses are being controlled in order to maintain the balanced immune system. In the present study, SLP was found one of the important lipoprotein regulating these responses during infection. The fat induced SLP along with infection have been seen significantly reducing the induction of pro-inflammatory responses. The proinflammatory cytokines especially IFN- $\gamma$ and TNF- $\alpha$ were found less induced in fat fed infected cells as compared to the infected one alone. Also these mononuclear cells upon 
culture in presence and absence of bacterial lysate together with SLP have been shown significantly reduction of these pro-inflammatory responses, but not from one cultured in presence of bacterial lysate only. These observations suggesting an important role for SLP in down-regulating cytokine expression, in other words down regulating the mononuclear lymphocyte proliferation. The mechanism by which surfactant lipids inhibit lymphocyte proliferation is not known. Since SLP is known to be produced by enterocytes upon giving dietary fat. There are studies that show that the dietary lipids are important in the modulation of immune/ inflammatory response [23] in various tissues. There is also well-documented evidence that fats modulate $\mathrm{T}$ lymphocyte activation [24]. Not only this, fish oil has been found to attenuate inflammatory responses partially via down regulation of T-lymphocyte function possibly by manipulating of intracellular signaling.

The mechanism of action of SLP isolated from the small intestine on cytokine regulation is largely unknown because the study is in its preliminary stage. Several studies have been conducted on the pulmonary surfactant and their immunoregulatory effects while the surfactant like particles from the intestine have not been studied in detail. Hence we have referred to studies conducted upon pulmonary surfactant.

In one of the studies conducted on the lung surfactants it has been observed that they have inhibitory effects on lymphocyte proliferation. Lymphocytes obtained from the lung lavage have long been known to be less responsive to mitogens than peripheral lymphocytes. The possibility that surfactant may be responsible for down regulating the lymphocyte function is supported by the finding that when peripheral lymphocytes were exposed to surfactant in vitro, their function was suppressed and they began to more closely resemble alveolar macrophages.

Our study highlights a similar kind of interaction between the SLP and the bacteria/ bacterial lysate. This in turn can affect the interaction between the host and the pathogen. The SLP may have an important say in this matter however, the type of fat used in the diet may be the deciding factor for infection in the intestines.

Further confirming the above finding, histological analysis have shown that microvillus damage in the cross section of the rat duodenum given infection while the microvilli from the fat fed infected group showed the shortening and broadening of villi only but not damage. This observation clearly indicates the fat induced SLP plays very important and protective role during infection.

In summary, we found that fat induced SLP decreases the level of infection possibly by preventing the colonization and translocation of Salmonella typhimurium. However, a detail of the mechanism has not been worked out. But our report gives pointers towards the lipoprotein as being important in controlling the infection. Further study is needed to trace out the mechanism of action of SLP and its possible therapeutic and antimicrobial use.

\section{Conflict of interest}

The authors have declared that no conflict of interest exists.

\section{References}

1. Lalmanach AC, Lantier F. Host cytokine response and resistance to Salmonella infection. Microbes Infect. 1999, 1:719-726

2. Gruenheid S, Finlay BB. Microbial pathogenesis and cytoskeletal function. Nature 2003, 422: 775-781

3. Rescigno M, Urbano M, Valzasnina B, et al. Dendritic cells express tight-junction proteins and penetrate the gut epithelial monolayers to sample bacteria. Nat Immun. 2000, 2:361-367

4. Niess JH, Brand S, Gu X, et al. CX3CR1-mediated dendritic cell access to the intestinal lumen and bacterial clearance. Science 2005, 307: 254-258

5. Henrik K, Takanori S, Bryan PH, et al. Salmonella enterica serovar Typhimurium regulates intercellular junction proteins and facilitates transepithelial neutrophil and bacterial passage. Am J Physiol Gastrointest Liver Physiol. 2007, 293: G178-G187

6. Mahmood A, Shao J, Alpers DH. Rat enterocytes secrete SLPs containing alkaline phosphatase and cubilin in response to corn oil feeding. Am J Physiol Gastrointest Liver Physiol. 2003, 285: G433-G441

7. Goetz GS, Mahmood A, Hultgren SJ et al. Binding of pili from uropathogenic E.coli to membrane secreted from colonocytes and enterocytes. Infect Immun. 1999, 67: 6161-6163

8. Eliakim RK, Alpers DH, Oren R et al. Abundance of surfactant-like particles reflects mucosal integrity in patients with peptic ulcer disease. Am J Physiol. 1996, 39:353-359

9. Lowry OH, Rose NJ, Farr AL et al. Protein measurements with the folin-phenol reagent. J Biol Chem. 1951, 193: 265-275

10. Zhang T, Stanely SL. Oral immunization with an attenuated strain of S.typhimurium expressing the serine rich Entamoeba histolytica protein induces antiamebic immune responses and protects gerbils from amebic liver abscess. Infect Immun. 1996, 64: 1526-1531

11. Powel DW, Plotkin GR, Maenza RM et al. Experimental diarrhea. Intestinal water and electrolyte transport in rat Salmonella enterocolitis. Gastroentrol. 1971, 60: 1053-1064

12. Davies MD, Parrot DM. Preparation and purification of lymphocytes from the epithelium and lamina propria of murine small intestine. Gut 1984, 22: 481-488

13. Nicole L, Brueton MJ. Intraepithelial, lamina propria and Peyer's patch lymphocytes of the rat small intestine: Isolation and characterization in terms of immunoglobulin markers and receptors for monoclonal antibodies. Immunology 1982, 45:775-783

14. Collet AJ. Preservation of alveolar type II pneumocyte lamellar bodies for electron microscopic studies. J Histochem Cytochem. 1979, 27: 989-996

15. Bao S, Beagley KW, France MP et al. Interferon gamma plays a critical role in intestinal immunity against S.typhimurium infection. Immunology. 2000,99: 464-472

16. Mahmood A, Engle MJ, Hultgreen SJ et al. Role of intestinal Surfactant Like Particles as a potential reservoir of uropathogenic E.coli. B B A. 2000,1523: 49-55

17. Sears LC. Molecular physiology and pathophysiology of tight junctions. V. Assault of the tight junctions by enteric pathogens. Am J Physiol Gastrointest Liver Physiol. 2000, 279: G1129-G1134

18. Adam O. Dietary fatty acids and immune reactions in synovial tissue. Eur J Med Res. 2003, 8: 381-387

19. Lugea A, Marisabel M, Ana AS et al. Epidermal growth factor increases surface hydrophobicity and resistance to acid in the rat 
duodenum. Am J Physiol Gastrointest Liver Physiol. 2001,43: G774 - G779

20. Lichtenberger LM, Romero JJ, Dial EJ. Surface phospholipids in gastric injury and protection when a selective cyclooxygenase-2 inhibitor (Coxib) is used in combination with aspirin. British Journal of Pharmacology 2007,150: 913-917

21. Haagsman HP. Interactions of surfactant protein $A$ with pathogens. Biochim Biophys Acta. 1998, 1408: 264-277

22. Bergman MA, Cummings LA, Alaniz RC, et al. CD4+-T-Cell Responses Generated during Murine Salmonella enterica Serovar Typhimurium Infection Are Directed towards Multiple Epitopes within the Natural Antigen FliC. Infect Immun. 2005, 73:7226-7235.

23. Cutler CW and Iacopino AM. Periodontal disease: links with serum lipid/triglyceride levels? Review and new data. J Int Acad Periodontal. 2003,5: 47-51

24. Yaqoob P. Lipids and immune response: from molecular mechanisms to clinical applications. Curr Opin Clin Nutr Metab Care. 2003,6:133-150 